\title{
旅京 \\ Electronic calibration of the ATLAS LAr calorimeter
}

Caroline Collard (LAL)

On behalf of the ATLAS Liquid Argon calorimeter group

Tipp09

Session Calorimeter IV : 14.03.09

IN2P3

stitut national de physique nucléaire

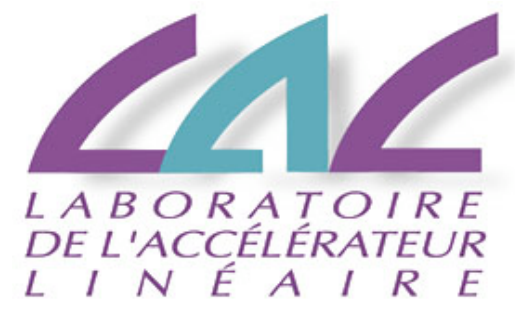




\section{Outline}

Description of the LAr calorimeters

Signal generation, readout and energy computation at cell level

Description of the calibration procedure :

- The calibration constants

- The chain to reconstruct them

- Their stability

LHC strategy running

Conclusions 


\section{The ATLAS LAr calorimeters}

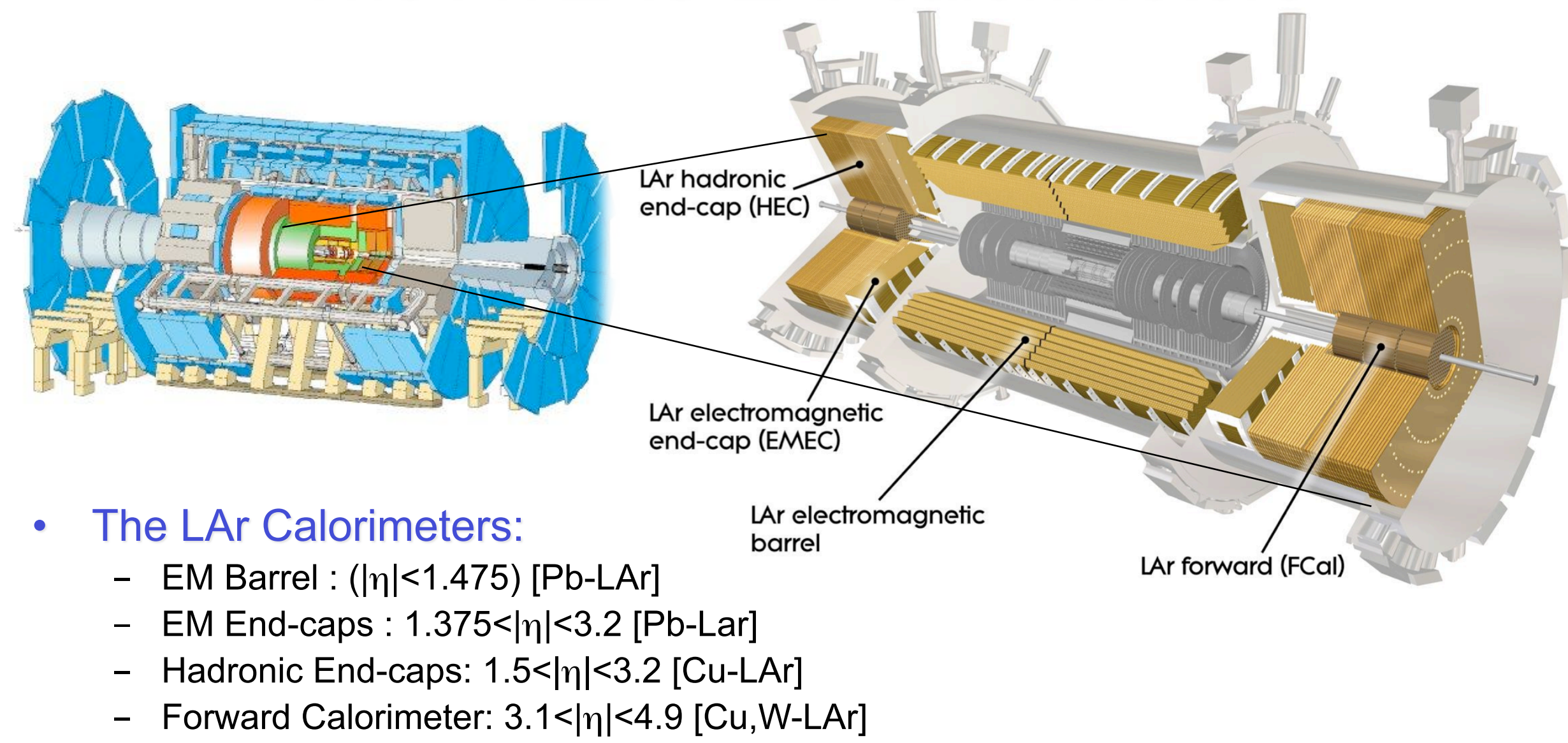

- are sampling calorimeters:

- LAr is the "active" medium

- Signal is the current generated by the ionization electron moving in the electrical field generated by the HV in the LAr "gap"

- ... and we have a lot ( 182k) of readout channels 


\section{The ATLAS Liquid Argon electromagnetic Calorimeter}

- 1 barrel

- 2 endcaps (inner \& outer wheels)

- Lead absorbers in a liquid argon bath at $88.5 \mathrm{~K}$

- Hermetic coverage in $\phi$ due to its accordion geometry

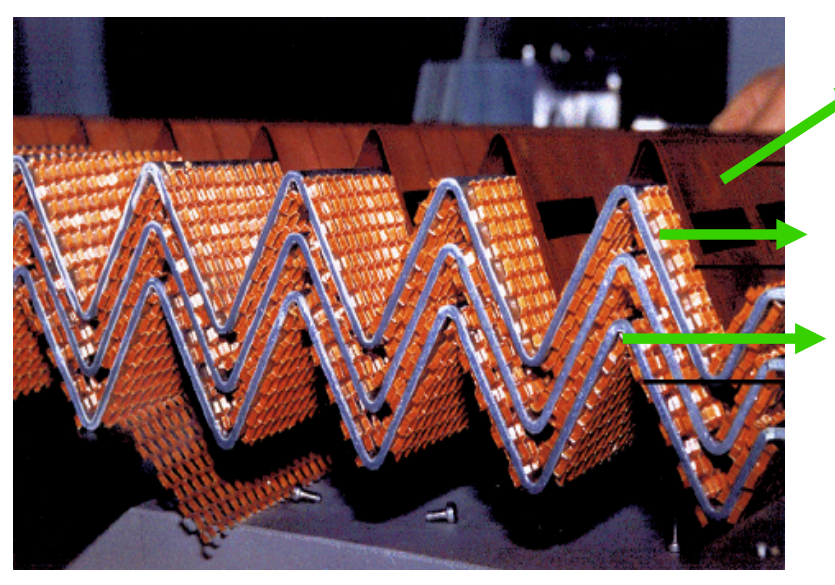

Cu/kapton electrode Honeycomb spacer

Steel-clad $\mathrm{Pb}$ absorber plates

- Longitudinal segmentation \& fine granularity: (for example in the barrel)

- S0 : presampler

- S1: front $\quad\left(\sim 4 X_{0}, \eta \times \phi: 0.025 / 8 \times 0.1\right) \eta=0$

- S2 : middle ( 16 $\left.X_{0}, \eta \times \phi: 0.025 \times 0.025\right)$

- S3 : back (2-12 Xo, $\eta \times \phi: 0.05 \times 0.025)$

• 173k channels in EM calorimeter
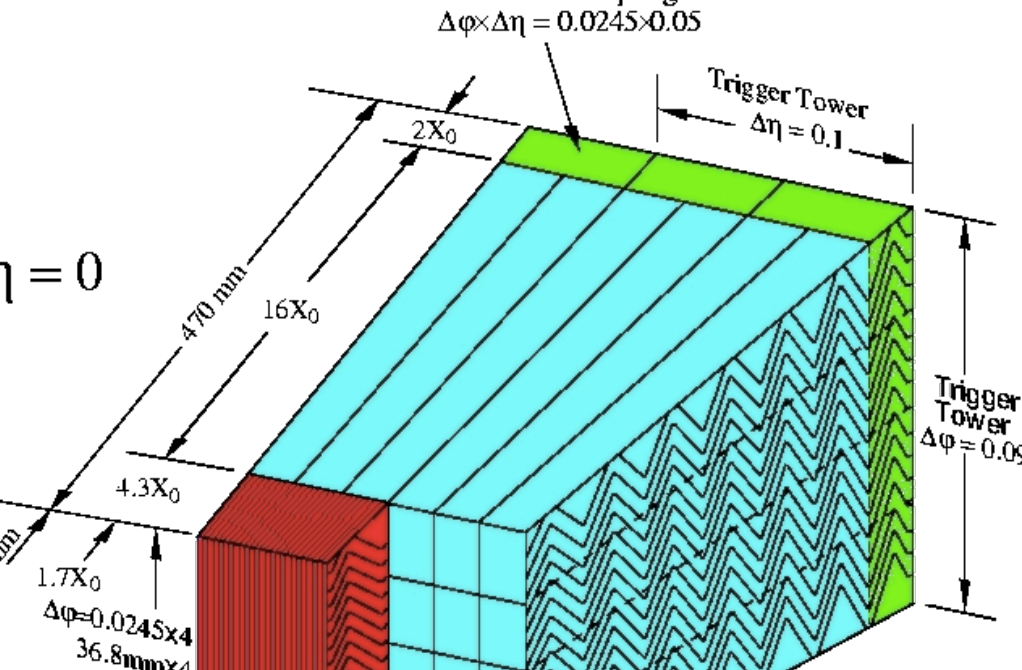
The ATLAS Forward and Hadronic Calorimeters

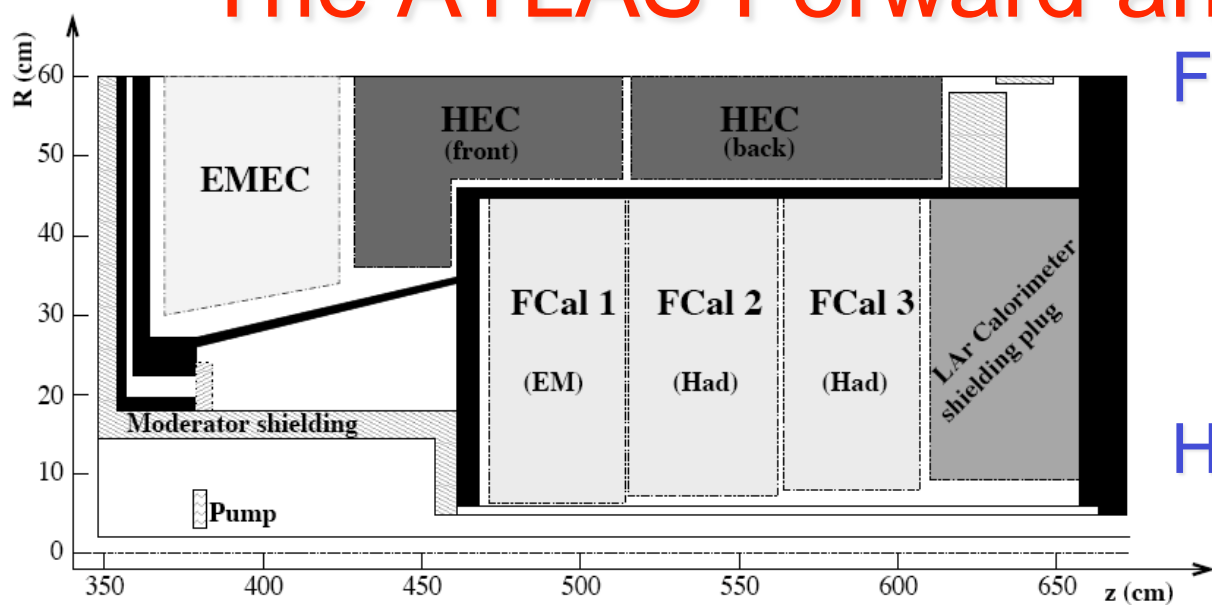

Forward Calorimeter (FCal) :

Per endcap : 1762 cells, 3 wheels $(10 \lambda)$

- Cu matrix for the first wheel $\left(2.6 \lambda, 28 X_{0}\right)$

- W matrix for the other two wheels $(2 \times 3.7 \lambda)$

- LAr gap FCAL 1/2/3: 250/375/500 $\mu \mathrm{m}$

Hadronic Endcap Calorimeter (HEC) :

Per endcap : 2816 cells, 2 wheels $(10 \lambda)$

- Cu absorbers (25mm/50mm thick)

- Each gap consists of 4 sub gaps of $1.85 \mathrm{~mm}$
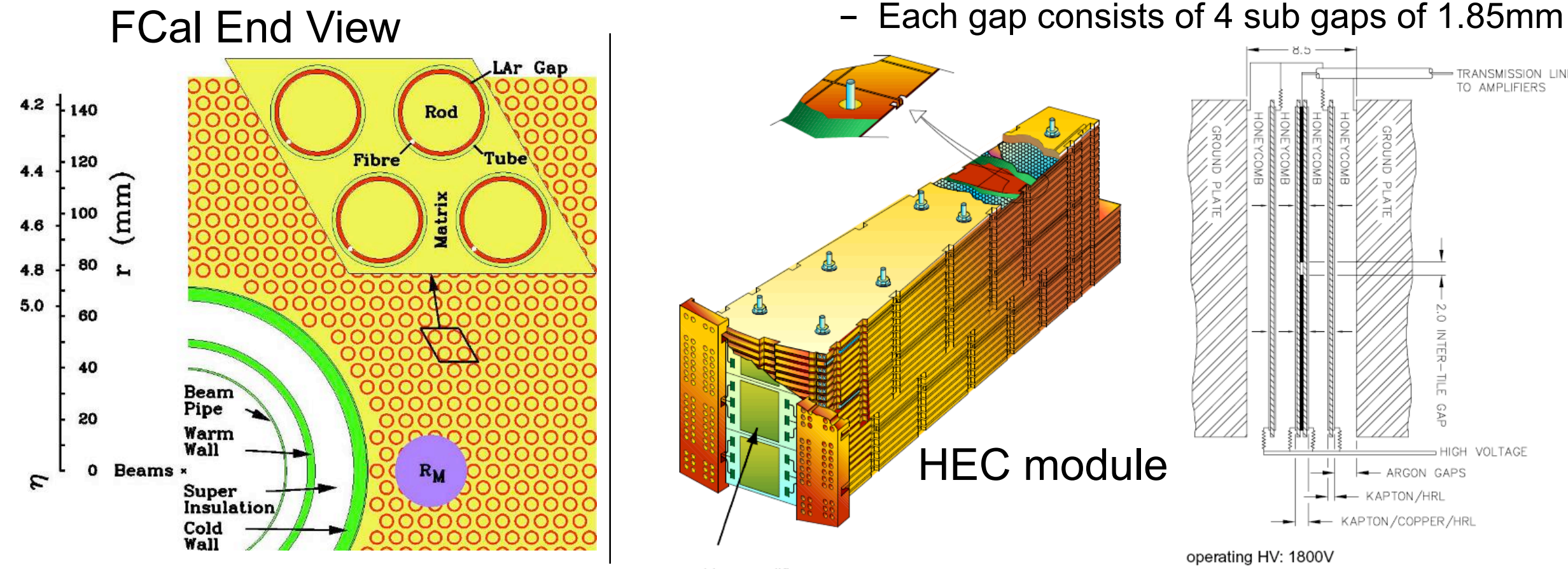

Since the geometry is different from the Electromagnetic Calorimeter, the ionization signals are slightly different... 


\section{Requirements on the EM calorimeters}

Large acceptance (accordion),

Particle identification (fine granularity \& longitudinal segmentation)

\section{Good energy resolution:}

- stochastic term : 10\%/ $\sqrt{\mathrm{GeV}}$

- noise term : $180 \mathrm{MeV}$ for EM clusters at low luminosity

- constant term : $0.7 \%$

$\rightarrow$ The electronics calibration plays an important role $(\sim 0.25 \%)$. It is used to obtain calibration constants for physics running (calibration runs in between LHC fills $\rightarrow$ constants used online for energy computation). In addition, it helps to find malfunctioning channels during commissioning.

[less stringent requirements on hadronic calorimeters :

HEC: $\sigma / E=50 \% / \sqrt{E}+3 \%$

FCal : $\sigma / E=100 \% / \sqrt{E}+10 \%]$ 


\section{From energy deposit to ADC counts}

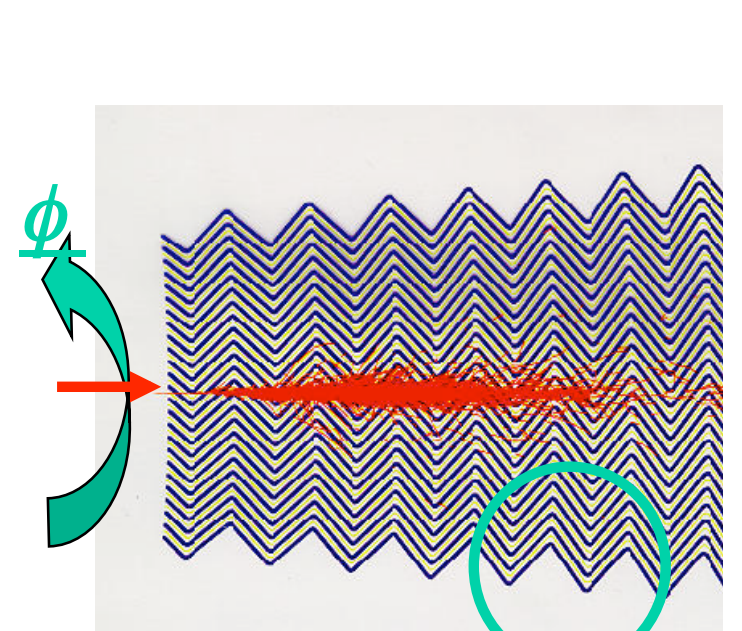

The LAr signal is generated by the ionization electrons drifting in the LAr gap thanks to HV between electrodes and absorbers. The peak of the ionization current is proportional to the energy released in LAr

Caroline Collard (LAL) outer copper layer inner copper layer kapton outer copper layer

stainless steel

glue
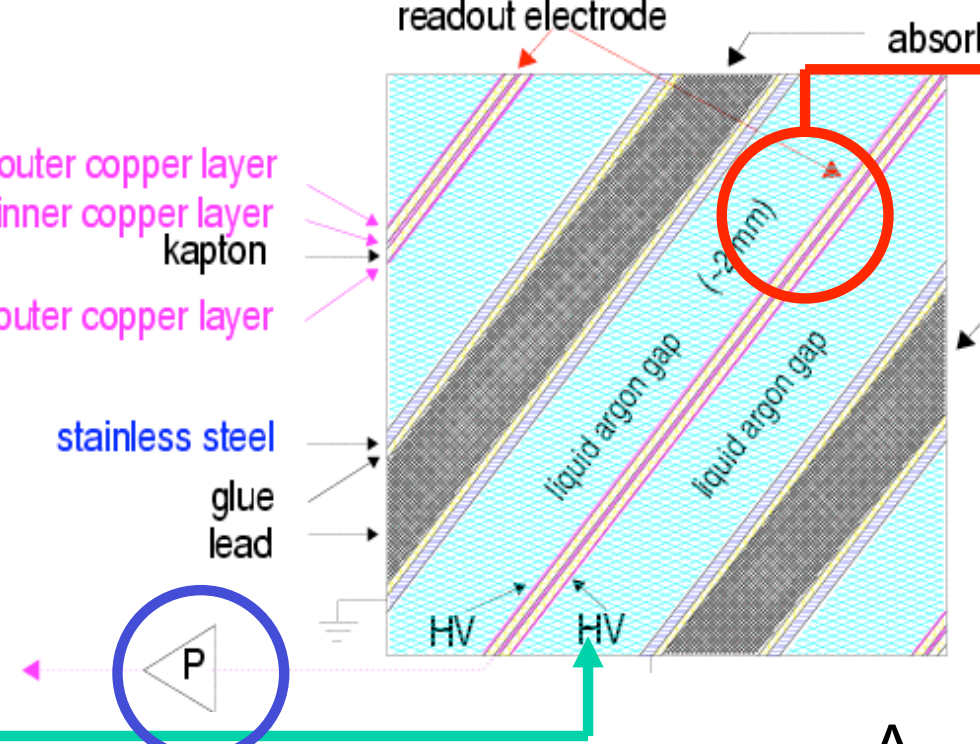

absorber

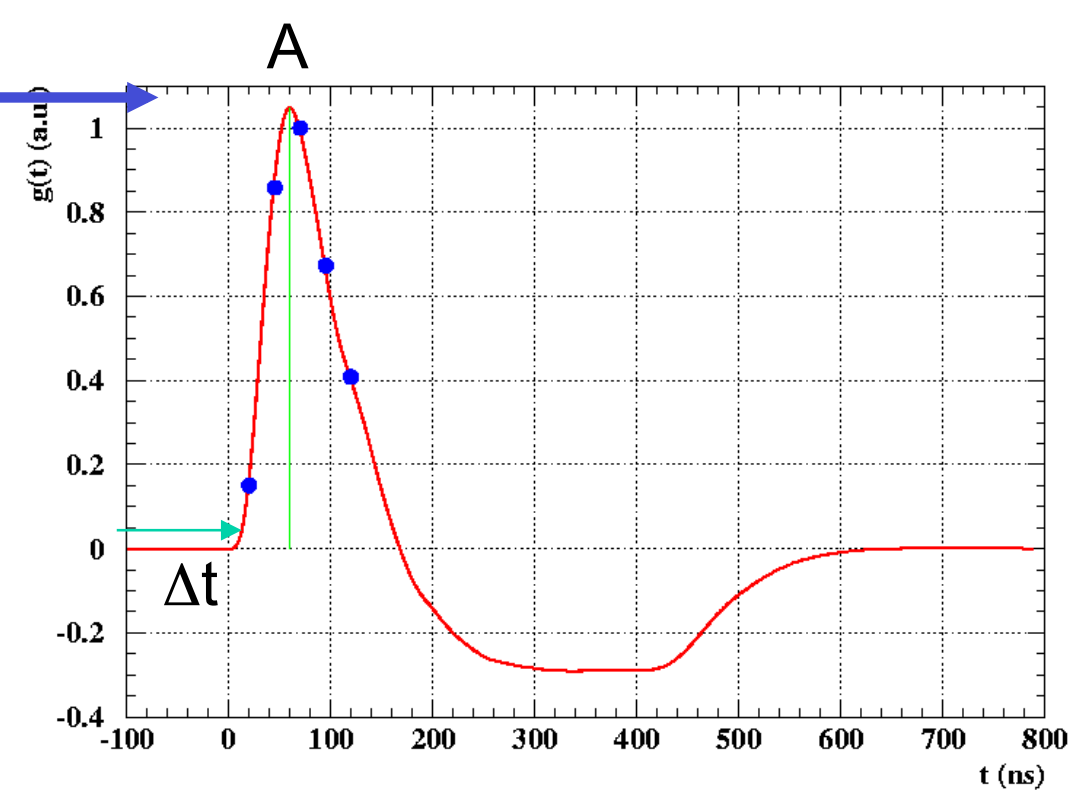

[5 samples in physics mode, up to 32 for calibration \& commissioning]
The triangular

current signal is pre-amplified and shaped (bipolar filter $\mathrm{CR}-\mathrm{RC}^{2}$ ), then sampled at the LHC bunch crossing frequency (every 25 ns) and digitized

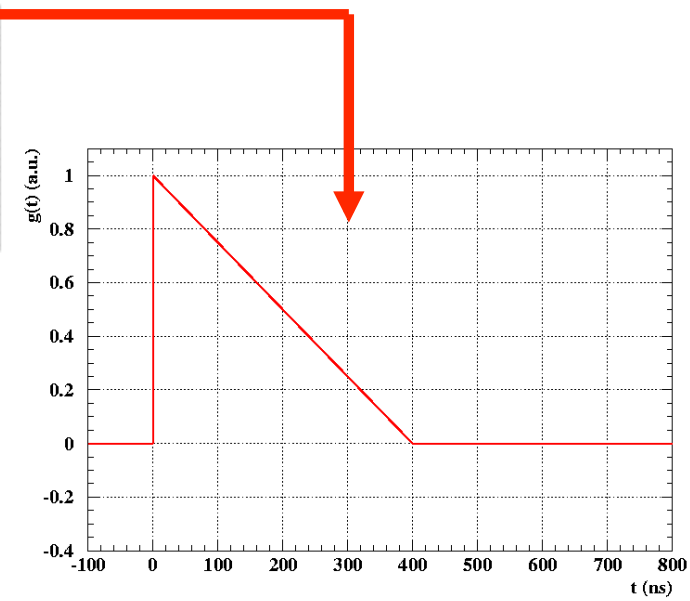




\section{Energy is computed online!}

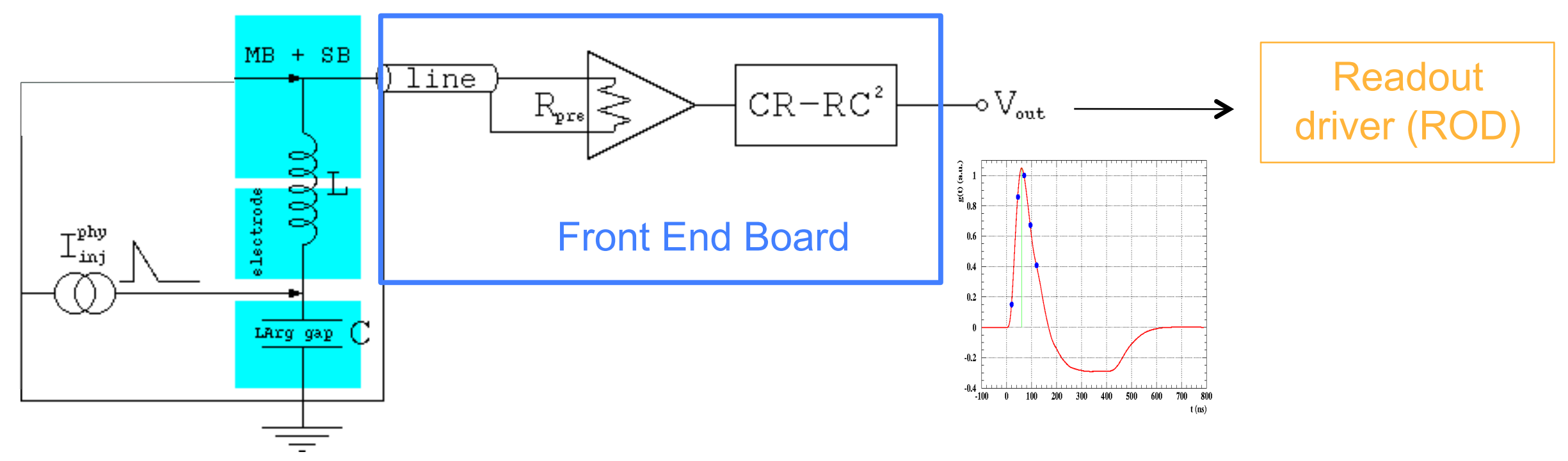

\section{Front End Boards on-detector:}

- Dynamical range: $30 \mathrm{MeV}$ to $3 \mathrm{TeV}$

-12bit ADC

- 3 different amplifiers : 100:10:1

- Bipolar shaping

- Analog storage during L1-trigger latency

- Digitization at $40 \mathrm{MHz}$

- Readout up to $75 \mathrm{kHz}$

\section{Readout drivers off-detector:}

- Calculate Energy, (and above threshold $E_{1}$ : Time and Quality) - Perform data-integrity checks \& higher-level monitoring

Above a certain threshold $E_{2}$, the 5 samples will be transmitted in addition to Energy, Time \& Quality 


\section{From ADC counts back to energy}
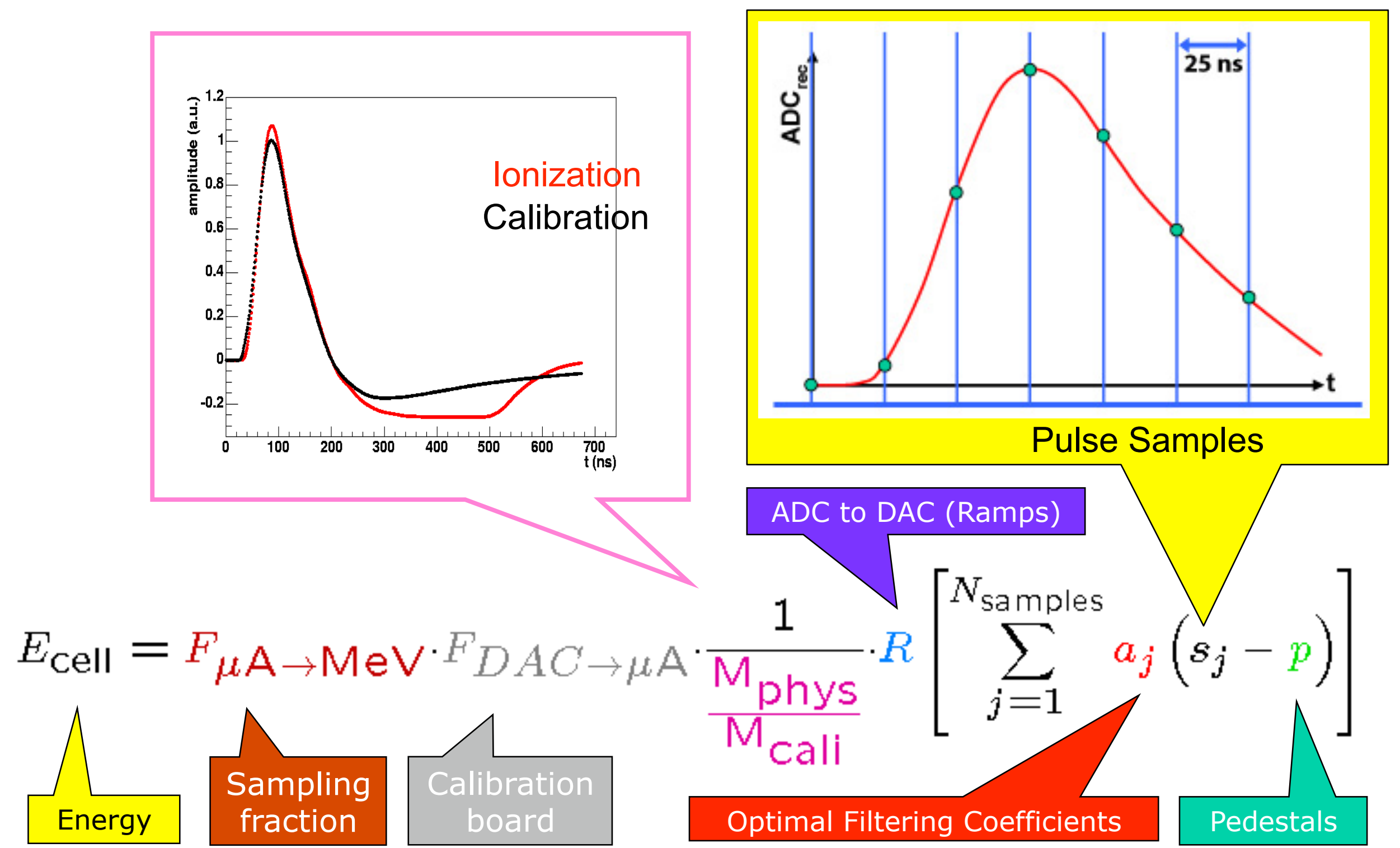


\section{The optimal filtering coefficients}

The Optimal Filtering (OF)

$$
\begin{aligned}
& \left\{\begin{array}{l}
\text { signal maximum amplitude } \\
\text { temporal position }
\end{array}\right. \\
& A_{\max }=\sum_{i=1}^{n} a_{i}\left(S_{i}-p\right) \Delta t=\frac{\sum_{i=1}^{n} b_{i}\left(S_{i}-p\right)}{A_{\max }}
\end{aligned}
$$

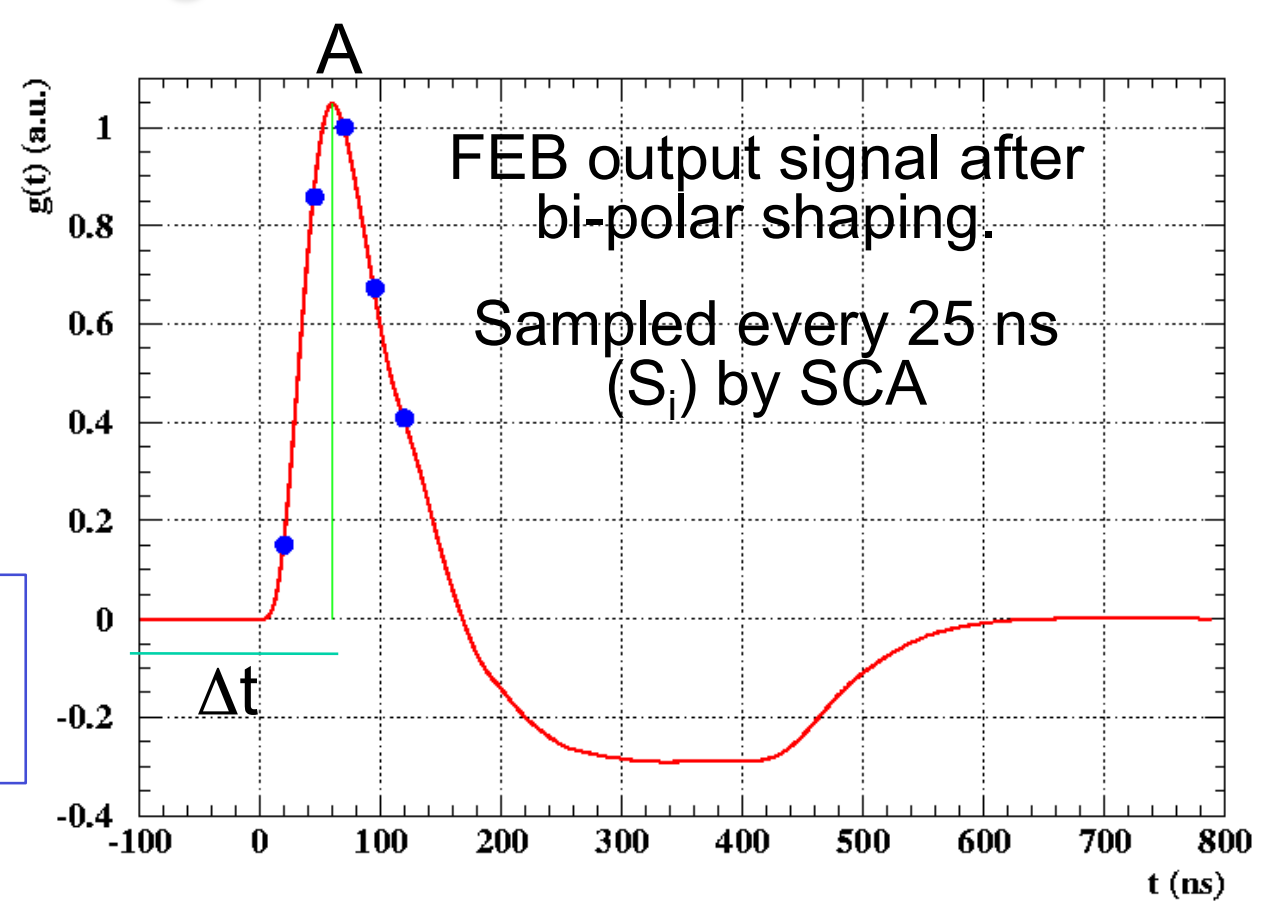

Optimal Filtering Coefficients (OFC), $\mathbf{a}_{\mathbf{i}}$ and $\mathbf{b}_{\mathbf{i}}$ are evaluated while minimizing the dispersion in $A_{\max }$ and $\Delta t$ arising from electronics and pile-up noise.

- OF is faster than a fit $\rightarrow$ important for online computation

- OF takes into account the time autocorrelation of noise

- Using 5 samples, the electronic noise is reduced by a factor $\sim 1.7$ with respect to a readout with only 1 sample

Need to know signal shape and the auto-correlation matrix for every cell 


\section{Calibration of the electronic response}

A known exponential current pulse is injected at the MB level and reconstructed through the full readout chain. The actual gain of each readout channel is computed.
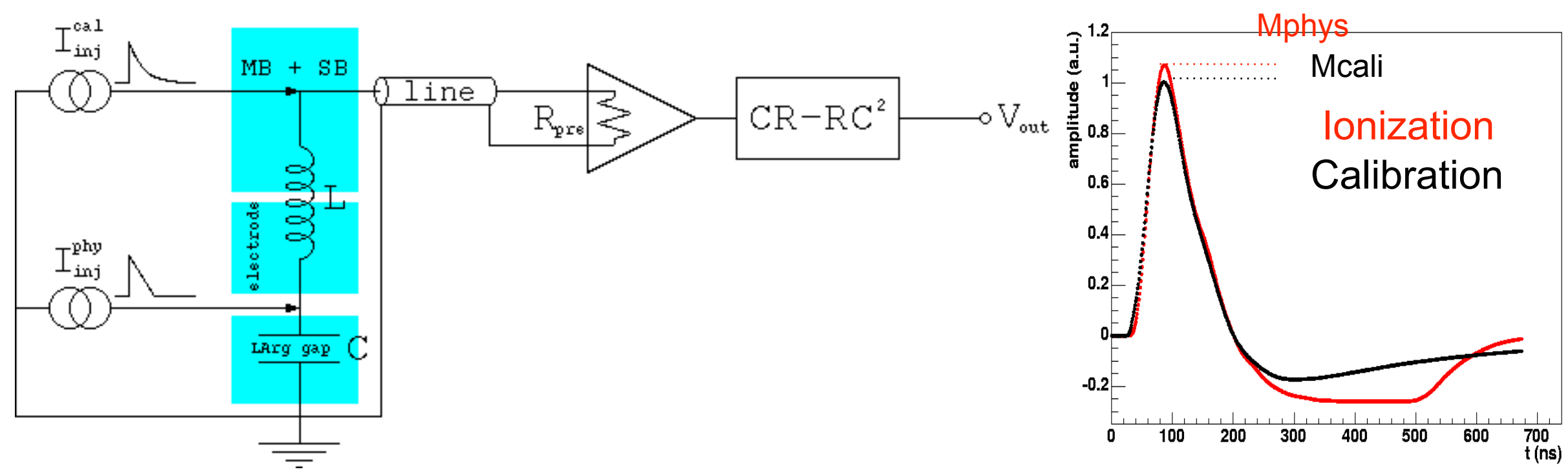

Calibration system installed on-detector:

- Charge injected very close to electrode.

- Dynamic range 16 bits.

- non linearity $<0.1 \%$.

The shaper output of the ionization and calibration signal corresponding to the same injected current is different! 


\section{Electronic calibration constants}
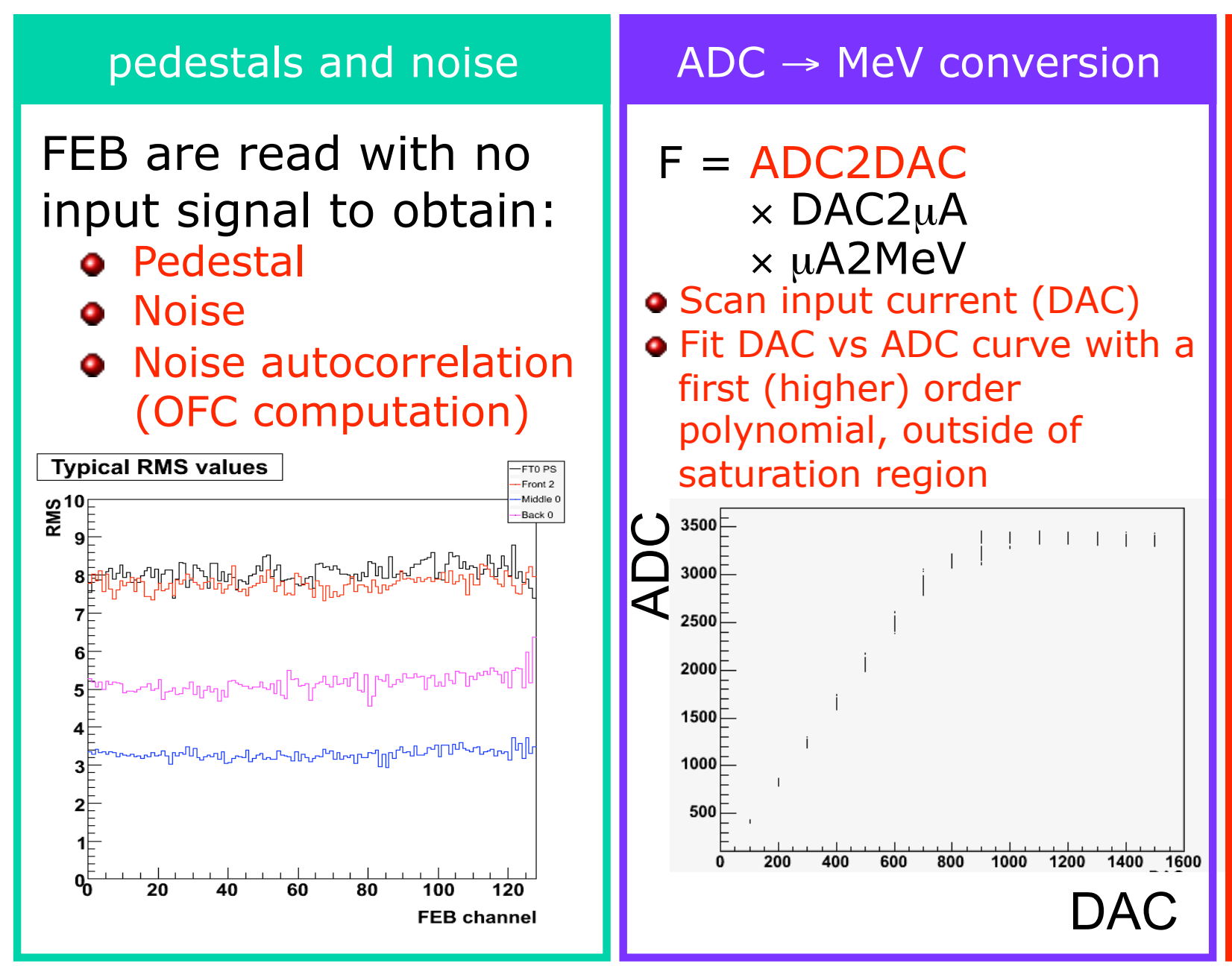

response to current pulse

All cells are pulsed with

a known current signal:

- A delay between

calibration pulses and

DAQ is introduced

- The full calibration

curve is reconstructed

$(\Delta \mathrm{t}=1.04 \mathrm{~ns})$

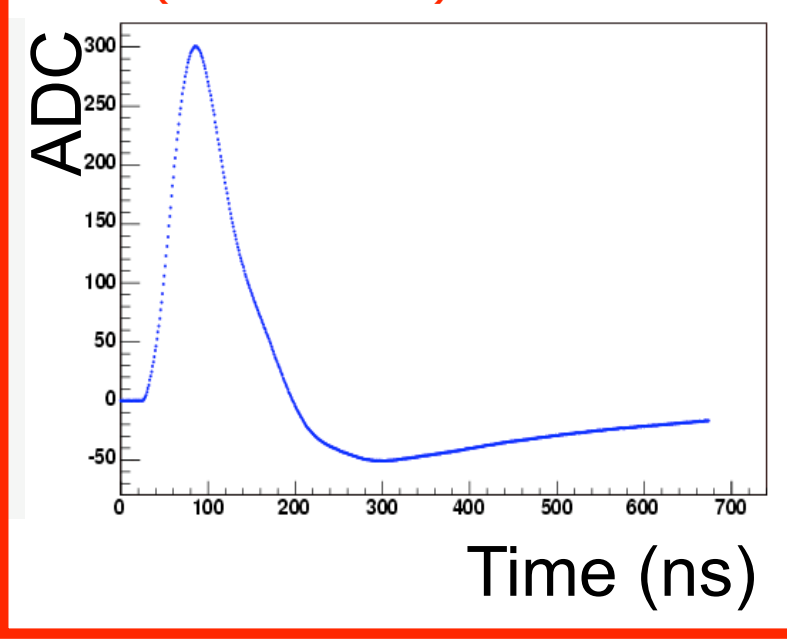




\section{Computation of the calibration constants}

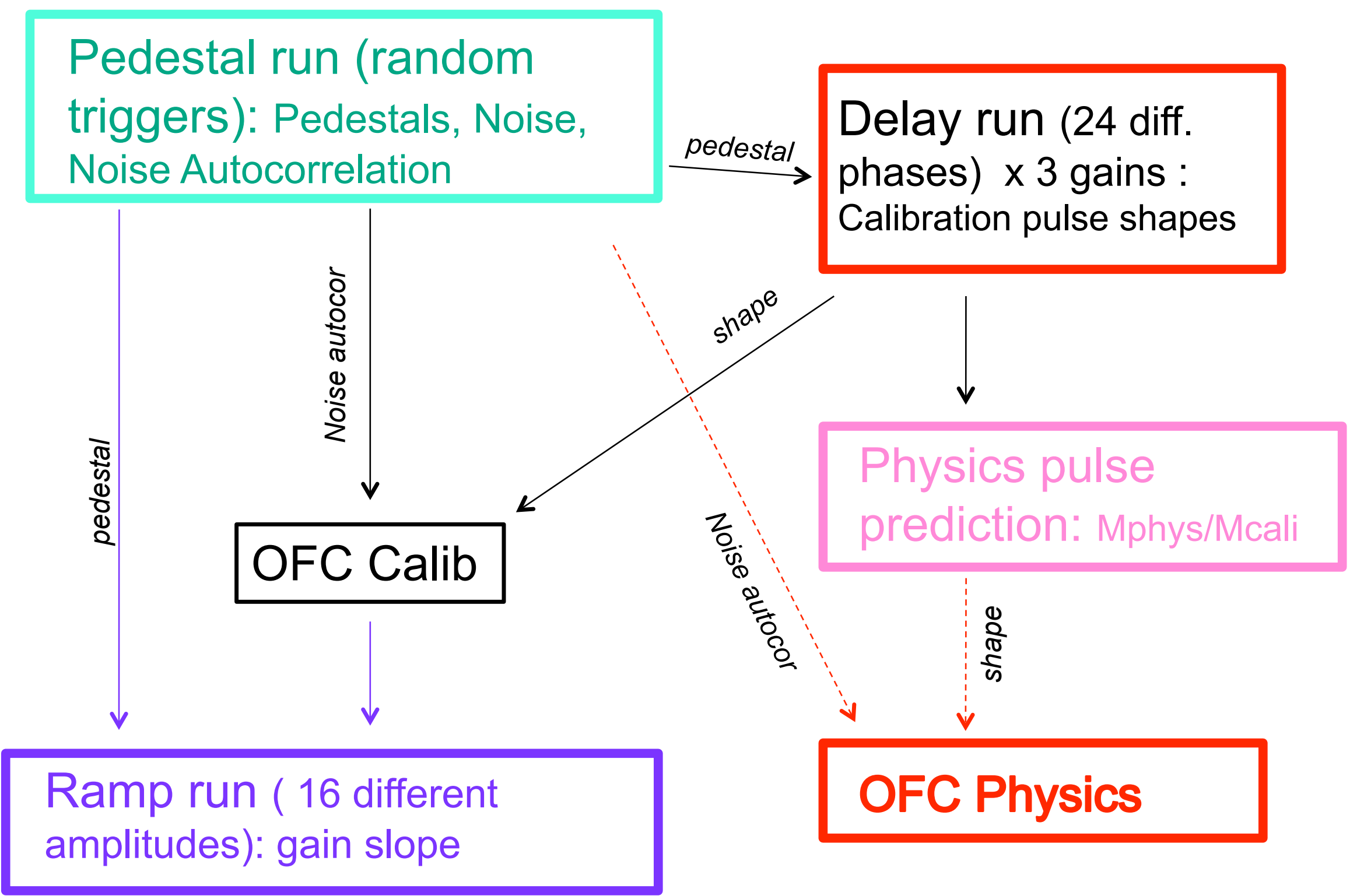




\section{Constant stability}

Take frequent sets of calibration data:

Pedestal-Gains-Pulses Automatic processing, validation vs reference and production of new constants ready for Data Base. Update it if needed.
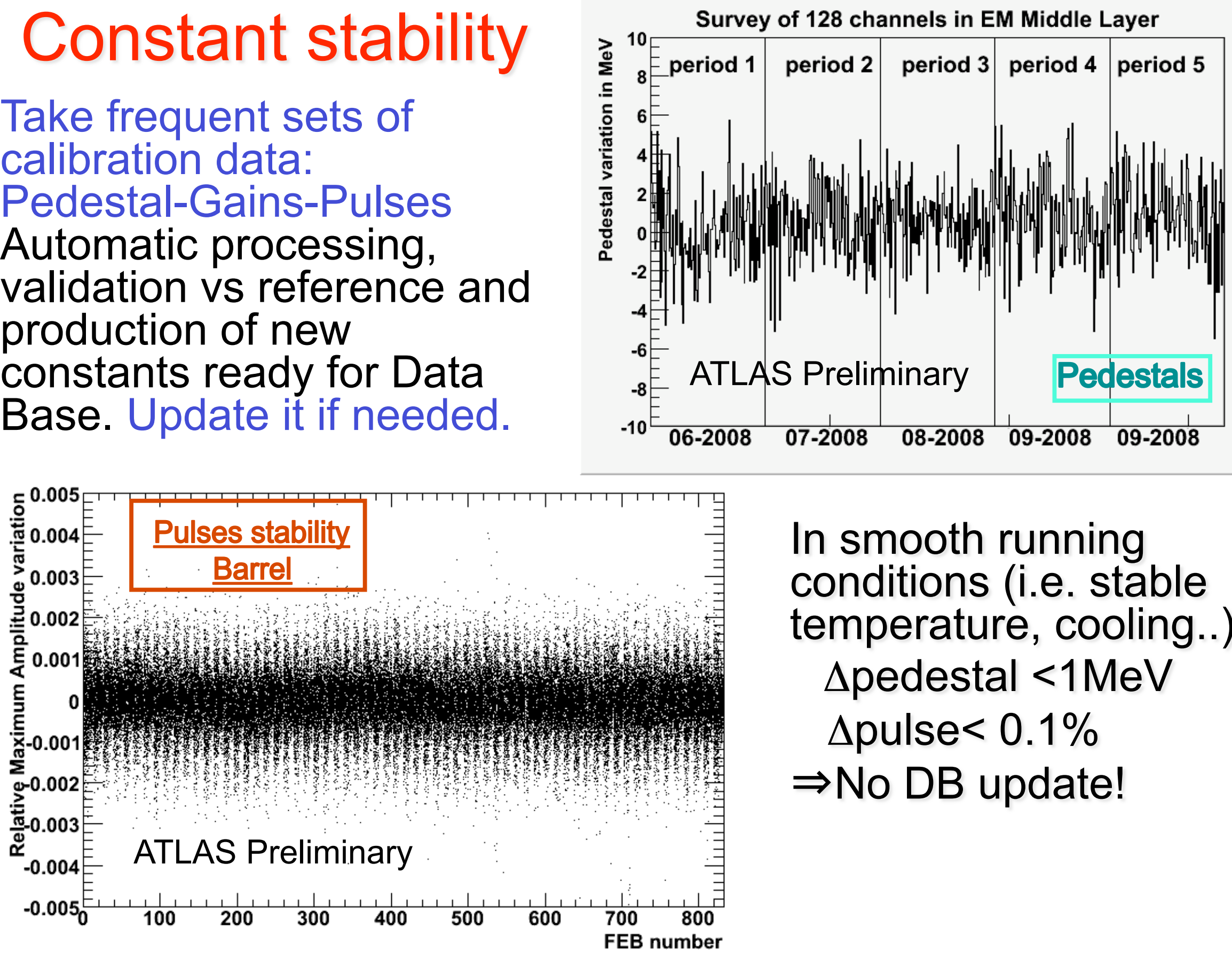

In smooth running conditions (i.e. stable temperature, cooling..) : $\Delta$ pedestal $<1 \mathrm{MeV}$ $\Delta$ pulse $<0.1 \%$ $\Rightarrow$ No DB update! 


\section{LHC running strategy}

At each LHC turnaround ( every $8 \mathrm{~h})$ :

- Take "pedestal" and "ramps" runs

- Reconstruct them and verify the stability

- If needed, upload the new condition constants into offline/online Data Base, take "Delay" run and compute the "OFC Physics"

If everything is stable, once per week:

- Check the stability of the calibration pulse shape, study the jitter

- Take special "pedestal" runs to study coherent noise

During physics runs:

- Monitor pedestal with random trigger

- Take special stream of data to monitor real ionization pulse

- Measure pileup + electronics autocorrelation matrix with minimum-bias event and/or random trigger 


\section{Conclusions}

- Calibration measurements (and cosmic muon data) are used successfully to commission LAr detector

- Complex formula to compute the energy of the cell :

- Used online

- Different ingredients mainly obtained from calibration runs

- Monitoring of the constants, stability expected

- Strategy for LHC running

- Electronics calibration is a key element to achieve a good energy resolution. 


\section{Back-up}




\section{"Problematic" channels}

$\cdot 0.02 \%$ dead or very noisy channels $\longrightarrow$ Masked in reconstruction

- $\sim 5 \%$ Channels with minor problems :

- increased noise

- damaged calibration lines

- $<6 \%$ of HV channels at reduced voltage, but sufficient voltage for usable signals in all cases

Effect on Drift Time and convertion $\mu \mathrm{A}->\mathrm{MeV}$ factor

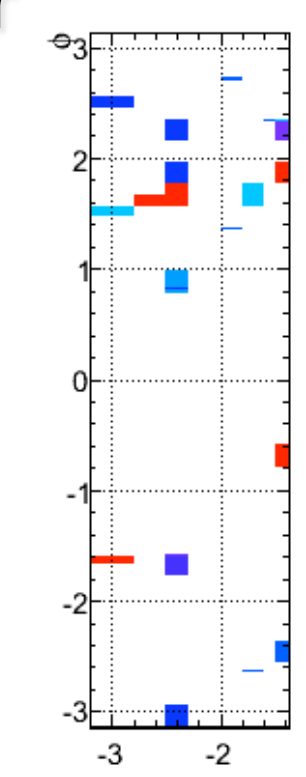

Tipp09
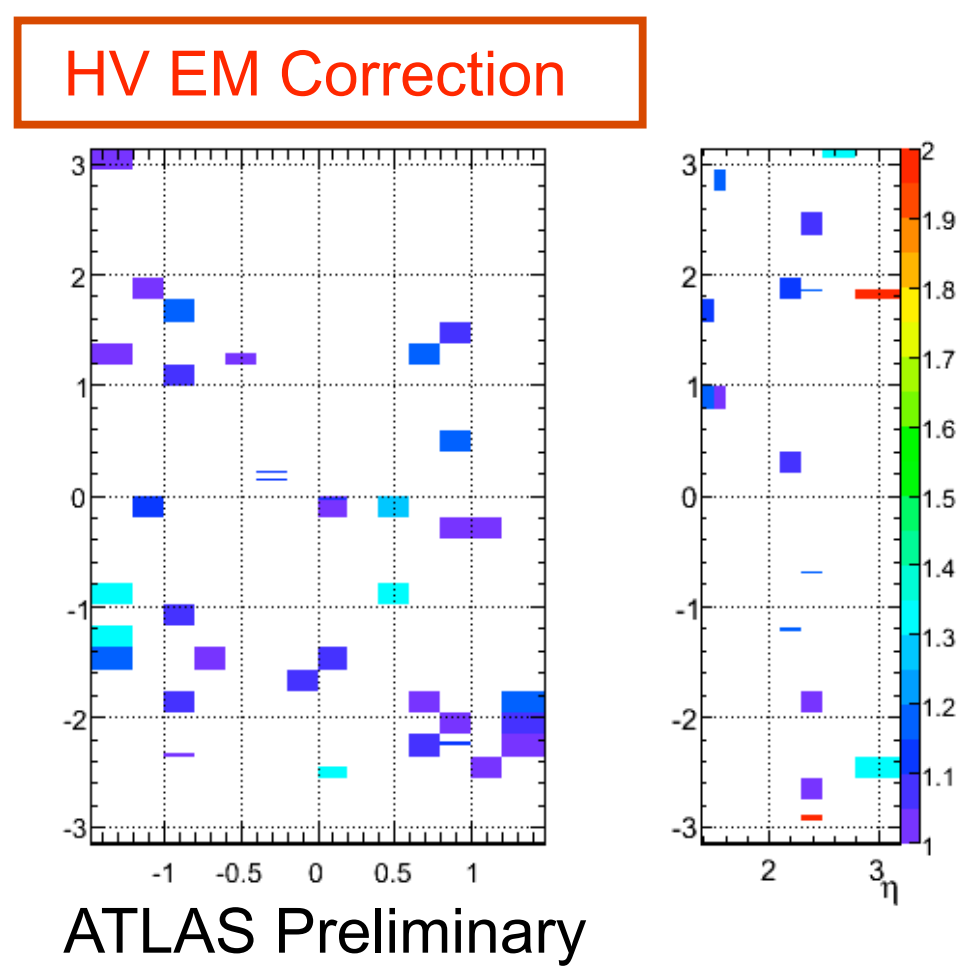


\section{The ATLAS Electromagnetic Calorimeter (EMC)}

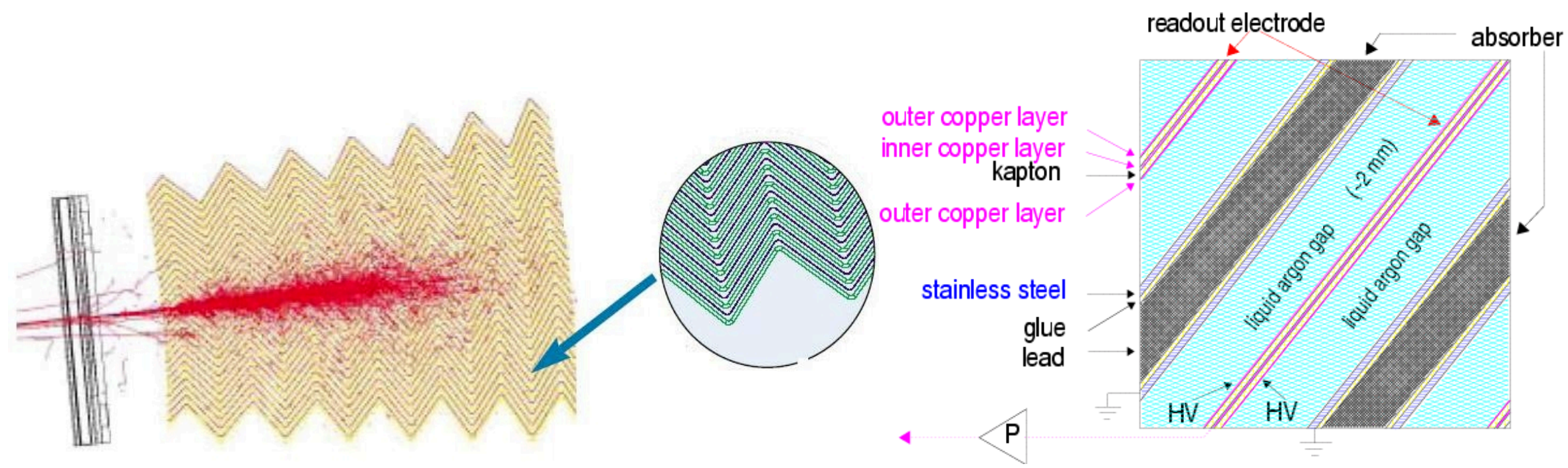

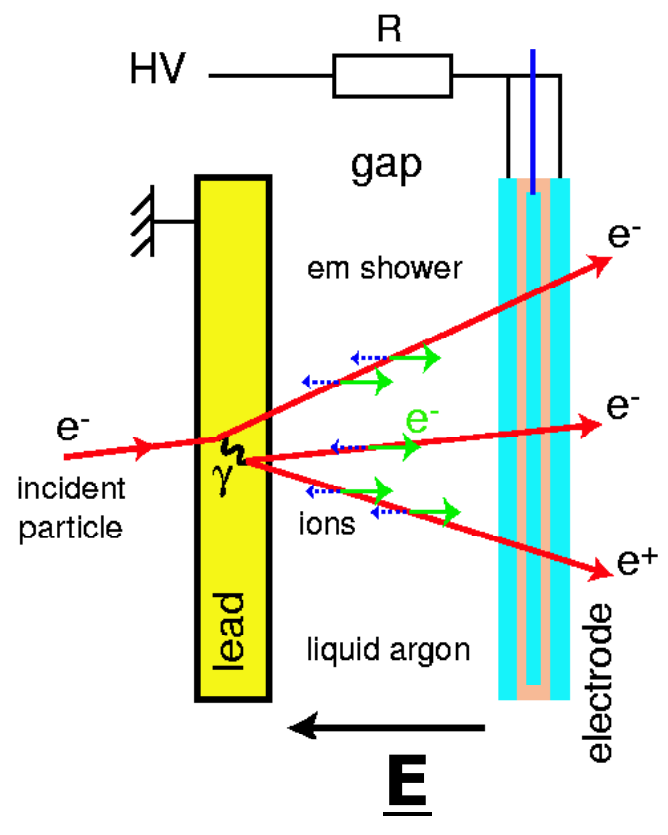

Caroline Collard (LAL) $\mathrm{i}(\mathrm{t})$

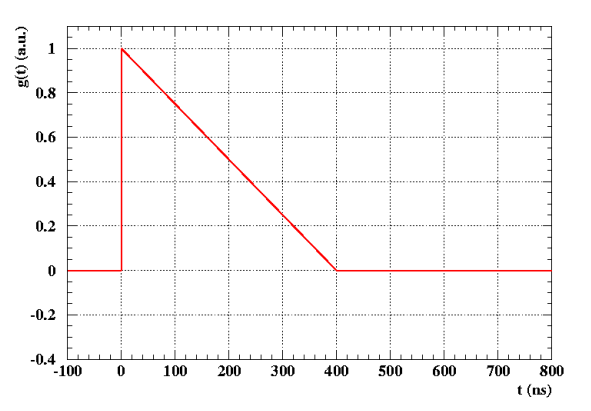

$\mathrm{t}_{\text {drift }}=\sim 450 \mathrm{~ns}$ in the EM, varies in the EMEC
- Accordion shape in EM barrel (EMB) and end cap EM calorimeters (EMEC)

$$
\checkmark>22 \mathrm{X}_{0}
$$

- 2 wheels (16 modules) in the EMB and 1 wheel (8 modules) per EMEC

- Main advantages

$\checkmark$ LAr as active material inherently linear

$\checkmark$ Hermetic coverage (no cracks)

$\checkmark$ Longitudinal segmentation

$\checkmark$ High granularity (Cu etching)

$\checkmark$ Inherently radiation hard

$\checkmark$ Fast readout possible 


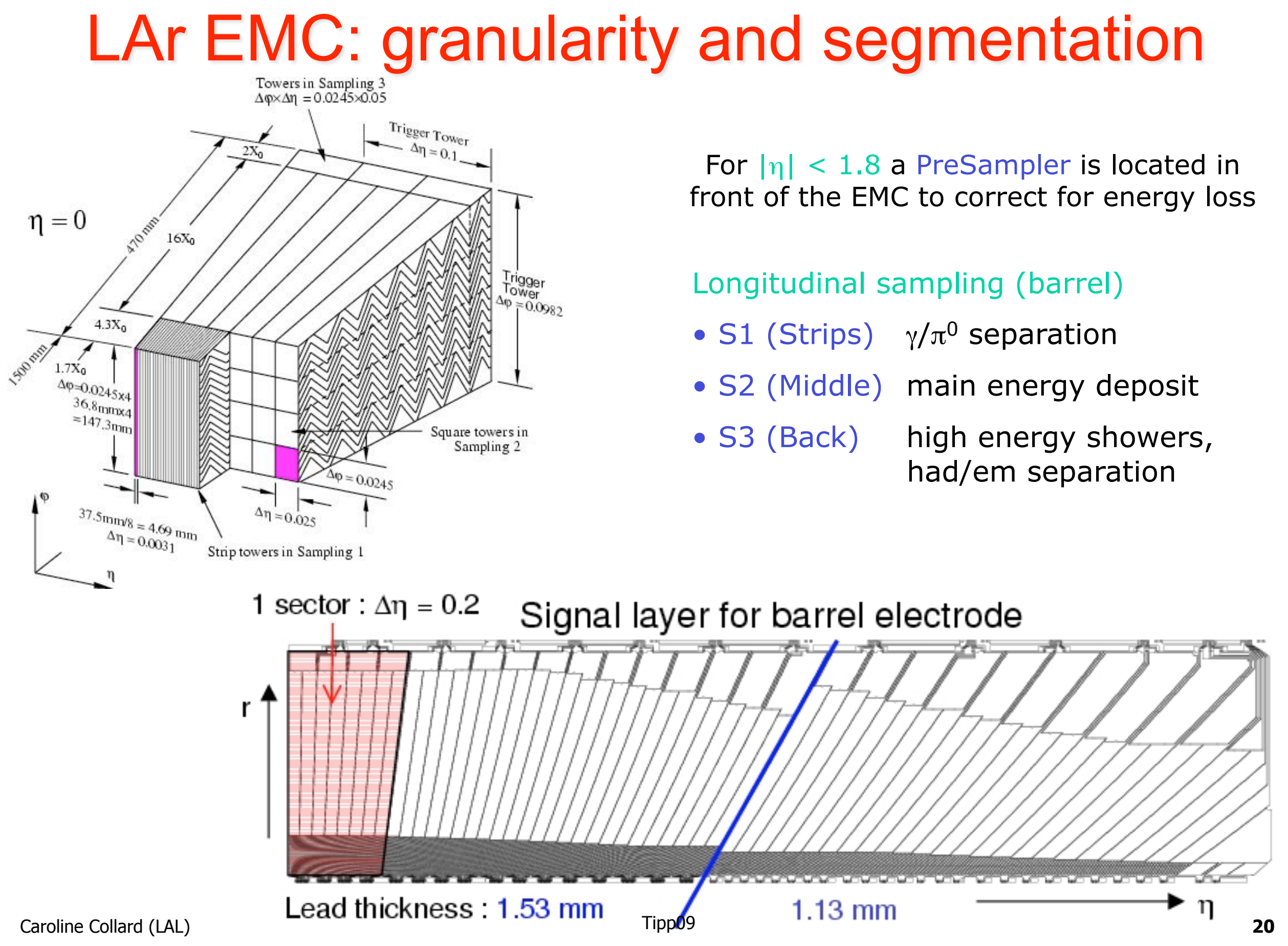


ATL- LARG-PUB-2007-010

\section{Xtalk in the strips}

Data, calculation
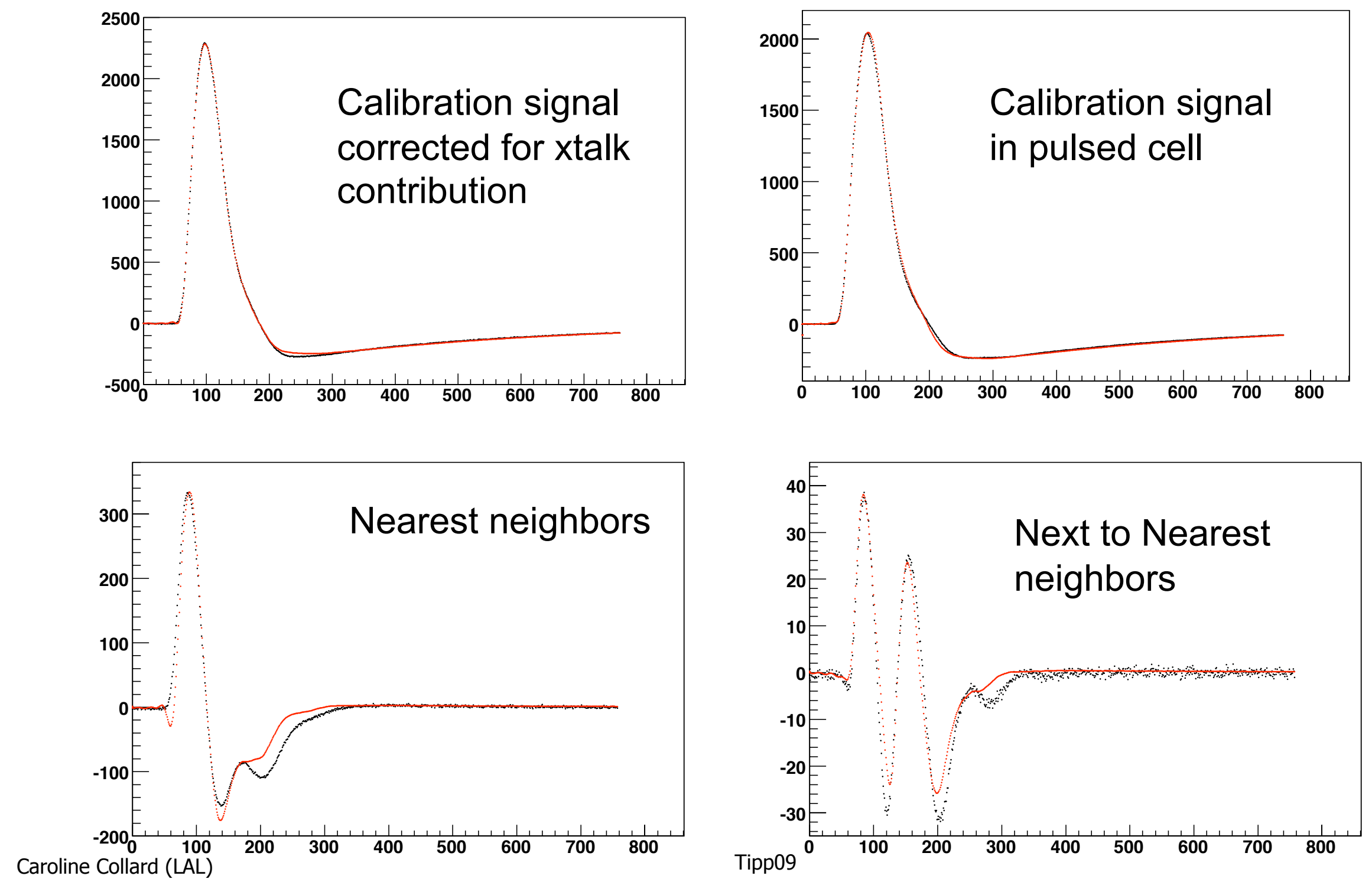


\section{LAr readout electronics}

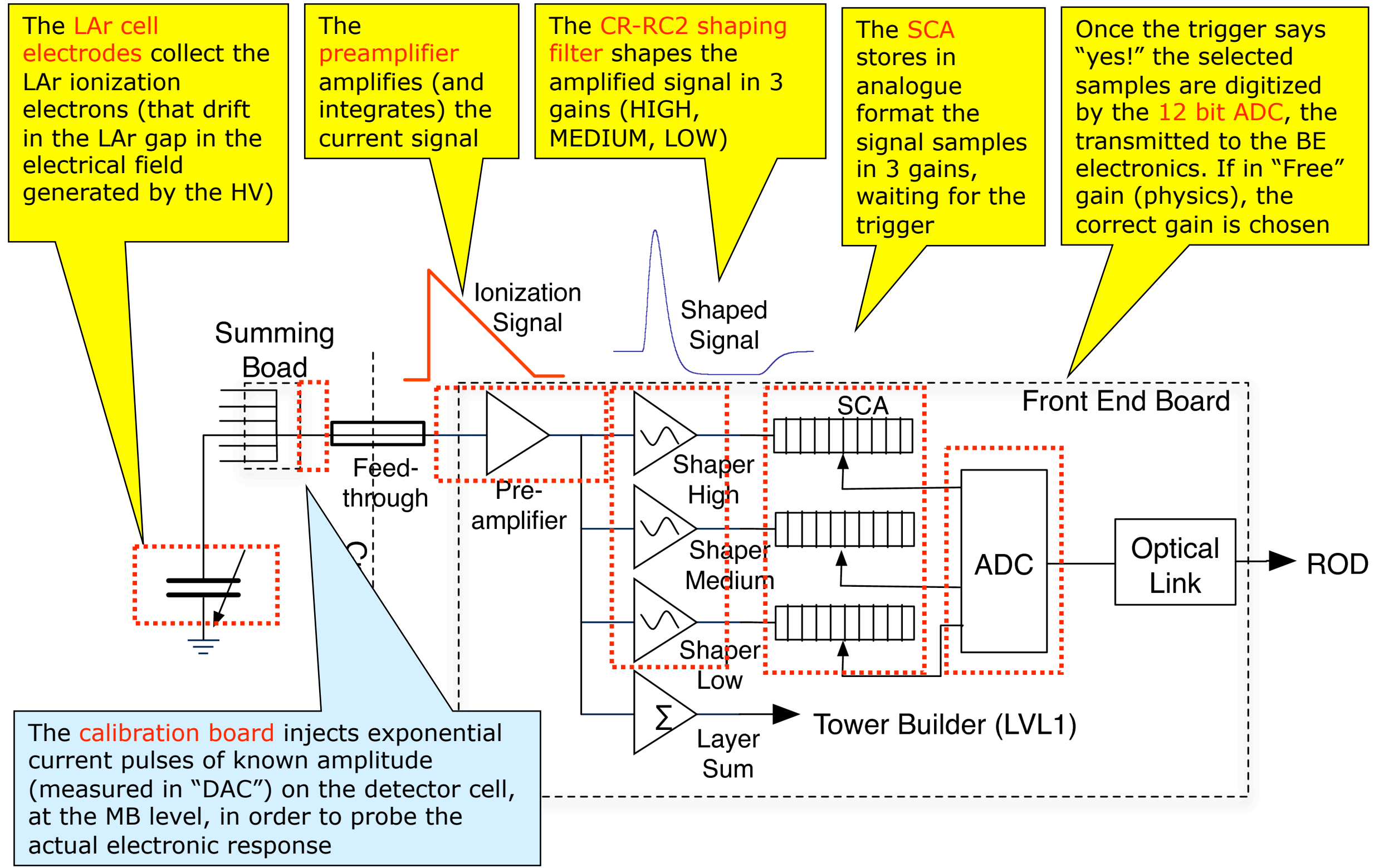




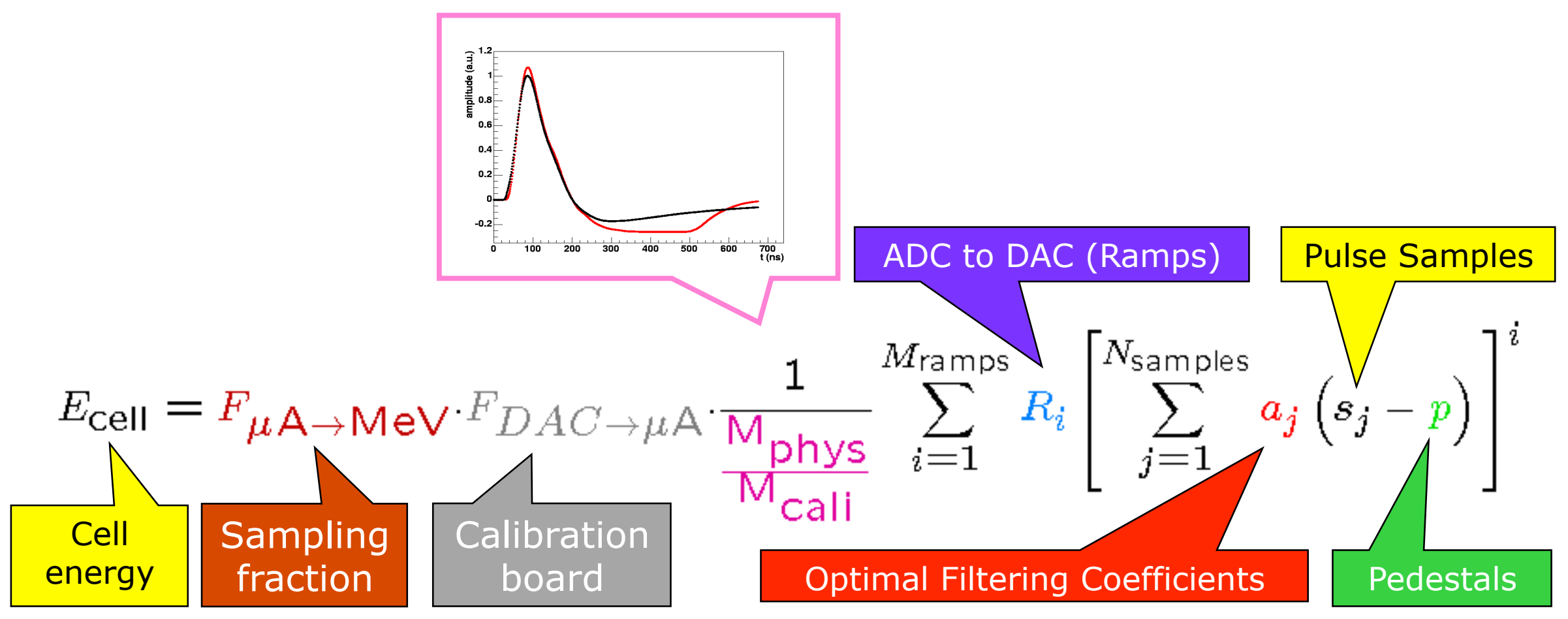

The above formula describe the LAr electronic calibration chain (from the signal ADC samples to the raw energy in the cell. Note that this version of the formula uses the general $\mathrm{M}_{\text {ramps }}$-order polynomial fit of the ramps. Actually we just use a linear fit (electronic is very linear, and additionally we only want to apply a linear gain in the DSP in order to be able to undo it offline, and apply a more refined calibration). In this case, the formula is simply:

$$
E_{\text {Cell }}=F_{\mu \mathrm{A} \rightarrow \mathrm{MeV}} \cdot F_{D A C \rightarrow \mu \mathrm{A}} \cdot \frac{1}{\frac{\mathrm{M}_{\text {phys }}}{\mathrm{M}_{\text {cali }}}} \cdot R\left[\sum_{j=1}^{N_{\text {samples }}} a_{j}\left(s_{j}-p\right)\right]
$$




\section{List of the ingredients}

\section{Pedestals}

$\checkmark$ Computed from a Pedestal run, it is the average of the triggers at a given sample...

Noise

$\checkmark$ Computed from a Pedestal run, it is the RMS of the triggers at a given sample...

- Used in topological clustering...

Noise Autocorrelation

$\checkmark$ Computed from a Pedestal run, it is the correlation of the different samples...

- Can come from electronics and/or pileup

Ramps

$\checkmark$ Computed from a Ramp run, measure the gain slope...

$\checkmark$ Ramp reconstruction needs the Pedestal to be subtracted, to be read from DB

$\checkmark$ Ramp reconstruction needs the Calibration OFC to reconstruct signal peak corresponding to given DAC

uA2MeV

- Calibration OFC are computed from Delay pulses and electronic noise Autocorrelation

$\checkmark$ Obtained from simulation

DAC2uA

$\checkmark$ Contains the $\mathrm{R}_{\text {inj }}$ value...

Calibration pulse shape

$\checkmark$ Obtained from a Delay run...

$\checkmark$ Delay reconstruction needs the Pedestal to be subtracted, to be read from DB

Physics pulse shape

$\checkmark$ May be predicted from the relative calibration pulse by several means...

Optimal Filtering Coefficients

$\checkmark$ Computed from:

- The "normalized" pulse shape (and derivative) for a given channel

- The noise autocorrelation matrix for a given channel 


\section{Prediction of the physics signal \\ RTM Method}

\section{"Factorization of the readout response"}

The readout response of each cell is probed

by the calibration pulses, and directly transferred to the physics pulse prediction

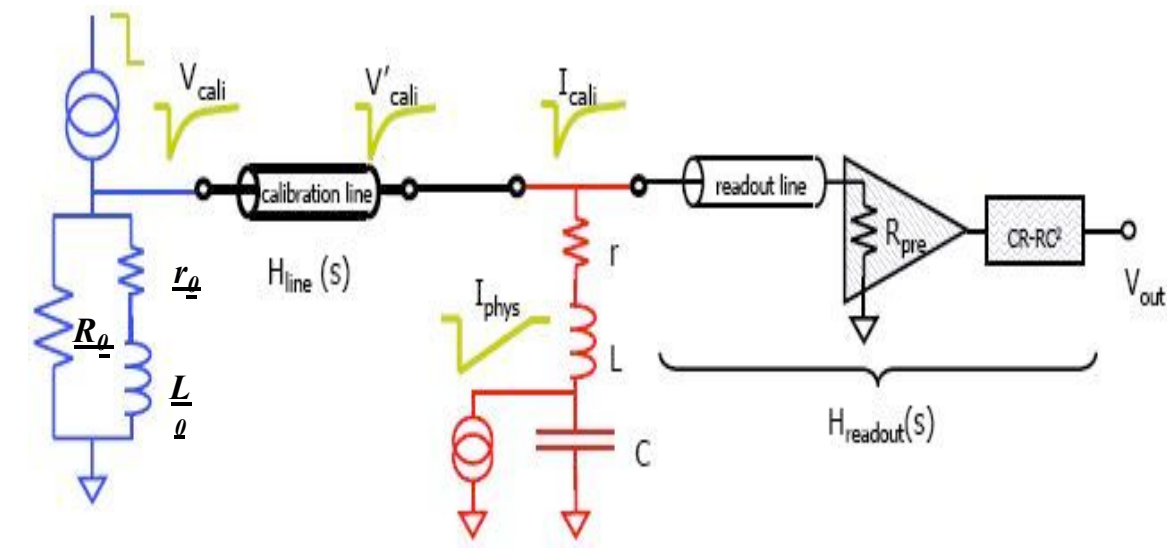

- The cell and pulse parameters ( $f_{\text {step }}, T_{\text {cali }}$, rC, LC) are completely obtained from the calibration pulses

- The only additional parameter required it $\mathrm{T}_{\text {drift }}$ (now from calculation, can be refined when enough data is collected)

$\rightarrow$ This method was successfully used in 2004 Test beam and is the default in the commissioning

\section{FPM Method}

"Analytical model of the readout response"

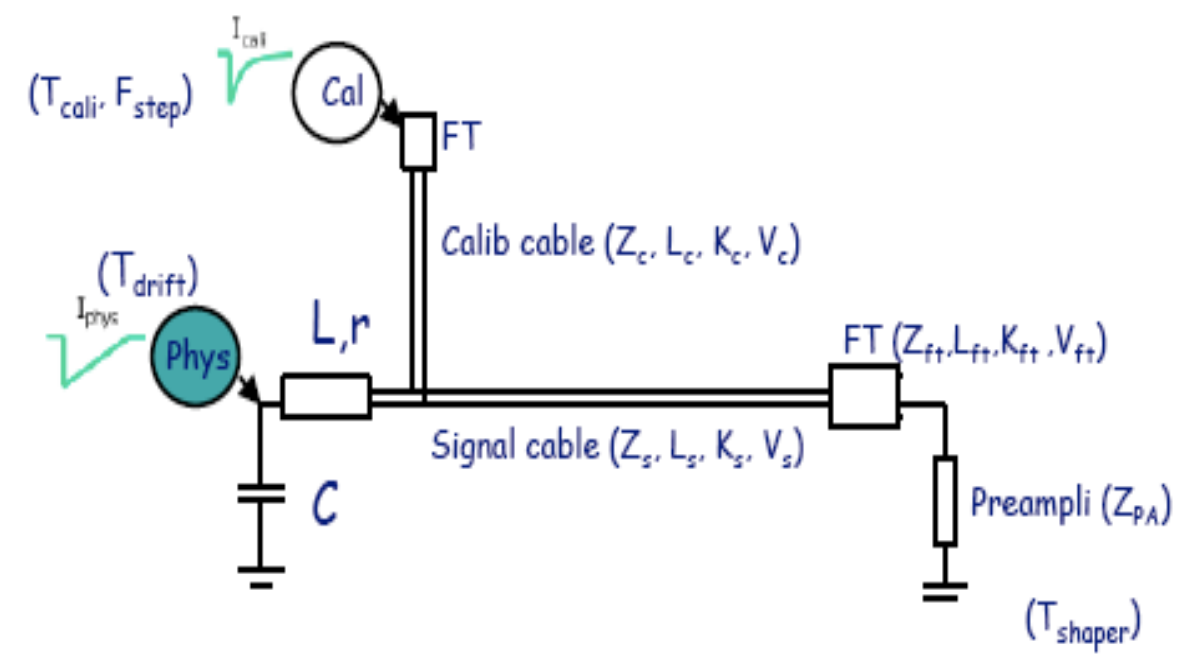

- Uses measured parameters where possible

- A few parameters $\left(T_{\text {shaper }}, Z_{s}\right)$ are left free to vary in order to match the measured calibration pulse response thus absorbing residual effects absent in the model

$\rightarrow$ Currently, available only in the barrel 